\title{
Use of Antidepressants in Older People during a 10-Year Period: An Observational Study on Prescribed Doses and Serum Levels
}

\author{
Kristine Tveit ${ }^{1}\left[\right.$ Monica Hermann ${ }^{1} \cdot$ Ragnhild Birkeland Waade $^{2}\left(\mathbb{D} \cdot\right.$ Roy Miodini Nilsen $^{1}$. \\ Susanna M. Wallerstedt ${ }^{3,4}$ - Espen Molden ${ }^{2,5}$
}

Published online: 20 July 2020

(c) The Author(s) 2020

\begin{abstract}
Background According to previous studies, older patients frequently have serum concentrations of antidepressant medication above the recommended reference range.

Objective The aim of this study was to investigate whether prescribed doses of antidepressants and the proportion of individuals with serum concentrations above the recommended reference range in older individuals ( $\geq 65$ years) have changed over a 10-year period in Norway.

Methods Serum concentration measurements and prescribed daily doses of antidepressants in 2007 and 2017 were extracted from a therapeutic drug monitoring (TDM) database at the Center for Psychopharmacology, Diakonhjemmet Hospital, Oslo, Norway. The database contains routine follow-up serum concentration measurements of psychotropic drugs for patients from all parts of the country. For citalopram, escitalopram, sertraline, mirtazapine and venlafaxine, the differences between 2007 and 2017 in mean prescribed doses and the proportion of patients with at least one serum concentration above the reference range, according to the Arbeitsgemeinschaft für Neuropsychopharmakologie und Pharmakopsychiatrie (AGNP) guidelines, were compared. For the proportion of patients with serum concentrations above the recommended reference range, differences between individuals aged $65-79$ and $\geq 80$ years were also examined.

Results The analyses of prescribed doses included 806 patients from 2007 and 1932 patients from 2017, with 972 and 2441 TDM samples, respectively. Between 2007 and 2017, modest reductions in prescribed daily doses were observed for citalopram ( $20 \mathrm{vs.} 17 \mathrm{mg} /$ day) and escitalopram (11 vs. $10 \mathrm{mg} /$ day), but the proportion of patients with serum concentrations above the recommended reference range was unchanged for both drugs, i.e. $11.5 \%$ vs. $12.4 \%$ for citalopram and $3.6 \%$ vs. $2.9 \%$ for escitalopram. For mirtazapine and venlafaxine, prescribed doses were reduced from 28 to $25 \mathrm{mg} /$ day and 150 to $125 \mathrm{mg}$ /day, respectively. A significant reduction in the proportion of individuals with serum concentrations above the recommended reference range was observed for mirtazapine $(27.1 \%$ vs. $11.5 \%)$ and for individuals aged $\geq 80$ years using venlafaxine (60.0\% vs. $30.0 \%)$. For sertraline, no differences in prescribed doses or serum concentrations above the recommended reference range were observed.

Conclusions Over a 10-year period, prescribed doses of antidepressants have been slightly reduced in older Norwegian patients, but a considerable proportion is still exposed to high serum concentrations of antidepressants.
\end{abstract}

Electronic supplementary material The online version of this article (https://doi.org/10.1007/s40266-020-00784-9) contains supplementary material, which is available to authorized users.

Kristine Tveit

kristine.tveit@hvl.no

Extended author information available on the last page of the article

\section{Introduction}

Depression is common among older people [1]. Antidepressant medication is a mainstay in the treatment of depression and the consumption of antidepressants among older individuals is high [2-4]. In 2018, $11 \%$ of Norwegian senior residents were dispensed an antidepressant drug, as opposed to $6 \%$ of the total population in Norway [2]. The use of antidepressants in Norway is average when compared with international statistics on drug consumption, and is approximately 


\section{Key Points}

Modest reductions in prescribed doses of antidepressants in older individuals were observed from 2007 to 2017, but the proportion of individuals with serum concentrations above the recommended reference range was only reduced for mirtazapine and for individuals aged $\geq 80$ years using venlafaxine.

For the most commonly used antidepressants citalopram and escitalopram, the proportion of individuals with serum concentrations above the recommended reference range was similar in 2017 and 2007, i.e. around 12\% and $3 \%$, respectively, despite reductions of $10-15 \%$ in the prescribed doses.

half of that in the United States [5, 6]. The prominent consumption raises questions regarding the benefit and safety of antidepressant medication in older individuals [2, 4, 7-9].

General treatment recommendations suggest cautious treatment of older individuals with psychotropic drugs because of an increased vulnerability towards drug effects, and also age-related pharmacokinetic alterations contributing to decreased elimination of many drugs [10-14]. Additionally, comorbidities and the number of drugs increase by age, and thereby the risk for drug interactions and adverse effects $[11,15]$. Altogether, comorbidity, polypharmacy, and changes in pharmacodynamics, as well as pharmacokinetics, make older individuals more prone to adverse effects, which can be especially harmful among older individuals [10, 11, 16]. At the same time, optimal treatment within the same age group may differ substantially because of larger differences in comorbidity and frailty with increased age [10, 17].

Increasing evidence of interindividual variability in serum concentrations of antidepressants exists, and these variations seem to increase in old age [18-21]. An earlier naturalistic study showed that serum concentrations of most antidepressants were 1.5- to 2-fold higher in individuals $>65$ years of age compared with younger individuals [18]. Additionally, the risk of serum concentrations above the upper level of the reference range was doubled among older individuals compared with younger persons [22]. These findings indicate that older individuals need lower antidepressant doses, compared with younger patients, to reach the same serum concentration. In the aforementioned study, the doses used by older individuals were somewhat reduced (10-30\%), but the general reduction in prescribed doses was not sufficient to compensate for the increased serum concentration among older individuals [18].

A national Patient Safety Campaign in Norway over the last decade has increased the focus on drug safety, including excessive use of psychotropic drugs among older individuals
[23]. Additionally, in 2011, relating to an increased awareness of a dose-dependent risk of QT-prolongation, new warnings, contraindications and recommendations on maximum doses were highlighted for both citalopram and escitalopram $[24,25]$. It may be speculated that the increased focus on cautious drug treatment in older patients, as well as more knowledge on adverse drug reactions, may have contributed to a reduction in prescribed doses and serum concentration levels of antidepressants in older individuals. However, as far as we are aware, this potential development in treatment with antidepressants over time has not previously been investigated.

The aim of this study was therefore to investigate whether prescribed doses of antidepressants and the proportion of individuals with measured serum concentrations above the recommended reference range have been reduced over a 10 -year period in older individuals, focusing on five frequently used antidepressants in Norway. Data from a therapeutic drug monitoring (TDM) service were used, since this provides information both on prescribed doses and actual serum concentration levels.

\section{Methods}

\subsection{Patients and Samples}

Data were acquired from a routine TDM database at the Center for Psychopharmacology, Diakonhjemmet Hospital, Oslo, Norway. The database contains serum concentration measurements of psychotropic drugs for patients from all parts of the country, both from primary and secondary care. TDM for the clinical follow-up of patients using newer-generation antidepressants is relatively common in Norway and is recommended by the AGNP, an interdisciplinary working group of TDM experts from German-speaking countries, established in order to promote the appropriate use of TDM in psychopharmacology [26].

The study included data on citalopram, escitalopram, sertraline, mirtazapine and venlafaxine, the five most frequently prescribed antidepressants in Norway when disregarding tricyclic antidepressants (TCAs), as TCAs are often used for indications such as pain relief. This decision was based on the ability to attain a sufficient sample size for comparisons between 2007 and 2017. To investigate whether any observed changes in prescribed doses and serum concentrations were specifically related to old age, data from individuals $<65$ years of age were also extracted from the database. Thus, all serum concentration measurements for patients aged 15 years and older treated with one or more of the aforementioned antidepressants in 2007 and 2017 were extracted from the database. Additionally, information on age, sex, prescribed drug dose and time between the last drug intake and blood sampling were recorded from the 
TDM files (requisition forms). Information on the use of multiple antidepressants was also registered. TDM samples lacking information on dose, age or sex were excluded.

\subsection{Drug Analyses}

Serum analyses of the antidepressants, including relevant metabolites, were performed by validated and certified analytical methods developed for routine TDM analyses at the Center for Psychopharmacology, Diakonhjemmet Hospital, Oslo, Norway. During the time span of the retrospective data collection, the analytical assays had been modified due to the renewal of the analytical instrumentation, but all modifications were cross-validated. Briefly, in the current ultraperformance liquid chromatography tandem mass spectrometry (UPLC-MS/MS) method, protein precipitation was first performed by mixing $500 \mu \mathrm{L}$ serum sample with $1000 \mu \mathrm{L}$ acetonitrile-methanol $(90 / 10 \mathrm{vol} / \mathrm{vol})$, centrifuged for $10 \mathrm{~min}$ at $1800 \mathrm{~g}$ at $-2{ }^{\circ} \mathrm{C}$, and $5 \mu \mathrm{L}$ of the purified serum samples were injected into an Acquity UPLC system (Waters, Milford, MA, USA) with a Micromass Quattro micro tandem MS detector (Waters) operated in electrospray ionizationpositive mode. Chromatographic separation of all compounds was performed on an Acquity UPLC BEH Shield RP18 column $(1.7 \mu \mathrm{m}, 1.0 \times 100 \mathrm{~mm}$; Waters $)$ by gradient elution from 18 to $45 \%$ acetonitrile in $10 \mathrm{mM}$ ammonium acetate buffer ( $\mathrm{pH} \mathrm{4.8)}$ in $2.8 \mathrm{~min}$. The total run time was $5.0 \mathrm{~min}$.

MS/MS detection was performed by multiple reactions monitoring at specific transitions for the different analytes and internal standards. Calibration curves $(n=5)$ ranging from $15-500 \mathrm{nM}$ for citalopram/escitalopram, 20-400 nM for mirtazapine, $20-300 \mathrm{nM}$ for sertraline, $100-2000 \mathrm{nM}$ for venlafaxine and 150-2000 nM for O-desmethylvenlafaxine, fitted to linear or quadratic polynomial functions, gave an $r^{2}$ of $>0.995$ for the different analytes. At both extremes of the calibration curves, the intra- and interday validation parameters of imprecision and inaccuracy showed $<15 \%$ deviation $(n=5)$ for all compounds. For venlafaxine, the serum concentration is given as the sum of the parent drug and the active metabolite.

\subsection{Data Analyses}

The data were separated into two age groups: patients aged $15-64$ years and patients aged $\geq 65$ years. In order to assess the changes in treatment with antidepressants among older patients between 2007 and 2017, the following data were calculated for patients $\geq 65$ years of age, for each of the antidepressants: (1) mean prescribed dose; (2) mean serum concentration; and (3) proportion of patients with at least one serum concentration above the upper level of the recommended reference ranges according to consensus guidelines determined by the AGNP [26]. As high serum concentrations may be more problematic in very old individuals, and may also differ between men and women, for (3) the proportions of patients with serum concentrations above the recommended reference range, differences between individuals aged 65-79 years and $\geq 80$ years, and sex differences, were also examined. For all endpoints, corresponding values were calculated for individuals $<65$ years of age to elucidate if any observed trends were related to old age or merely reflected trends irrespective of age.

For evaluation of mean serum concentrations, only samples obtained within $10-30 \mathrm{~h}$ after the last drug intake and with a detected serum concentration within the quantification limits were included, as serum concentrations outside these limits cannot be accurately determined (Fig. 1). However, samples above the upper quantification limits were included in the data analysis of the proportion of patients with serum concentrations above the recommended reference range (Fig. 1).

\subsection{Statistics}

Within the dataset, there were multiple samples from the same patients, and some patients had TDM measurements in both 2007 and 2017. To account for the dependency between observations from the same individual, linear mixed-effects models were used to analyze the differences in prescribed daily doses and serum concentrations between 2007 and 2017. Neither doses nor serum concentrations were normally distributed, and were therefore log transformed before analyses. For the same reason, data are presented as geometric means in 2007 and 2017. Sex was included as a covariate in the mixed-effects model of prescribed doses, whereas both sex and sampling time were included in the mixed-effects model of serum concentrations to adjust for possible biases related to sex and differences in the sampling time within the interval of 10-30 $\mathrm{h}$ between drug intake and time of the blood test.

For all five antidepressants, the proportion of individuals with at least one serum concentration measurement above the recommended reference range was determined in 2007 and 2017. Differences in the proportions of individuals with serum concentrations above the recommended reference range between 2007 and 2017, between older ( $\geq 65$ years) and younger (15-64 years) and young-old (65-79 years) and very old ( $\geq 80$ years) subjects, as well as sex differences, were evaluated using the Chi-square test. The Chi-square test was also used for the evaluation of use of multiple antidepressants in younger $(<65$ years $)$ individuals compared with older ( $\geq 65$ years) individuals.

All analyses of differences between 2007 and 2017 were performed for both older and younger individuals; the results for older individuals are presented in the main text, while the 


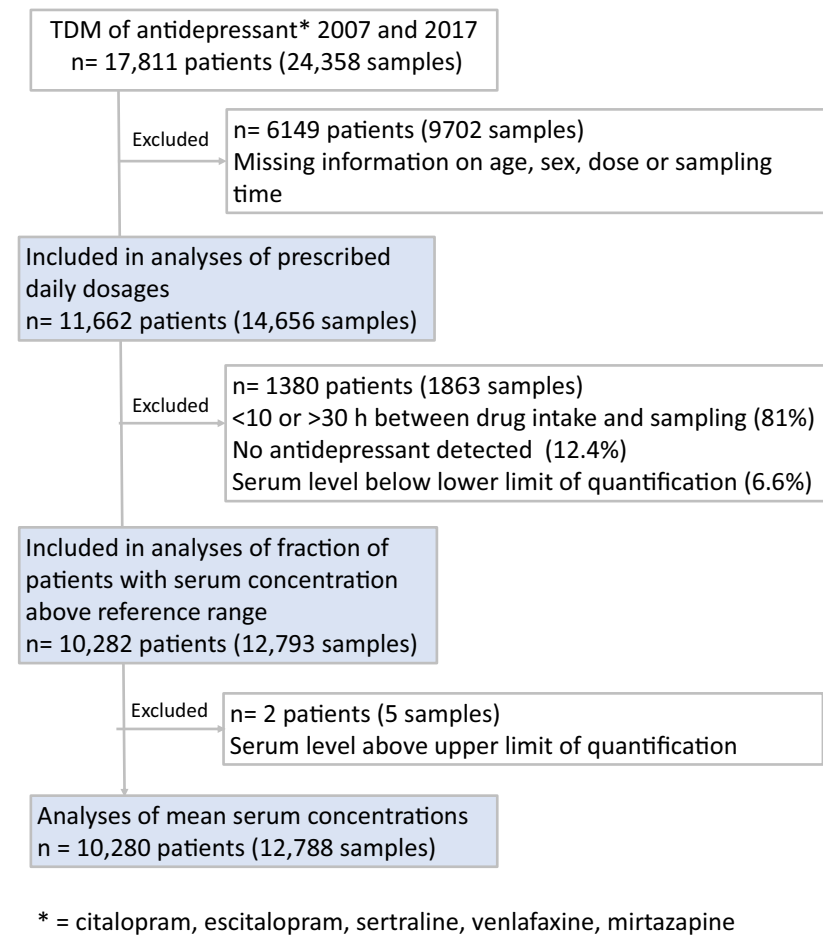

Fig. 1 Inclusions and exclusions of patients (samples). TDM therapeutic drug monitoring

results for younger individuals are presented in the online resources.

Differences in median age between 2007 and 2017 were calculated using the two-sample Wilcoxon rank-sum test, while differences in the proportions of females and older individuals were calculated using the Chi-square test.

Stata IC version 16 (StataCorp, LLC, Statistical Software, College Station, TX, USA) was used for statistical analyses. All tests were two-sided and $p$-values $<0.05$ were considered statistically significant.

\subsection{Ethical Considerations}

This study was approved by the Regional Committee for Medical and Health Research Ethics (ref. 2018/655) and the Hospital Investigational Review Board.

\section{Results}

\subsection{Included Patients and Samples}

Overall, 17,811 patients, regardless of age, with a total of 24,358 TDM analyses, were extracted from the TDM database (Fig. 1). Among these, 11,662 individuals (14,656 samples) were included in the analysis of dose trends, and
10,282 individuals $(12,793$ samples) were included in the analyses of the proportion of individuals with at least one serum concentration above the recommended reference range. Finally, 10,280 individuals (12,788 samples) fulfilled the criteria for analyses of difference in serum concentrations (Fig. 1). Approximately 20\% of the samples were from hospitalized patients, while the remaining samples were from outpatients or nursing home patients.

In our dataset, the total number of TDM samples of antidepressants in 2017 was higher than in 2007, and $15 \%$ of the samples were from older individuals in 2007 compared with $30 \%$ in 2017 (Table 1). The fractions of males and females were similar in 2007 and 2017 within both younger and older individuals (Table 1). For older individuals using escitalopram, mirtazapine and venlafaxine, median age was slightly higher in 2017 compared with 2007 (79 vs. 75 years, 82 vs. 77 years, and 75 vs. 72 years, respectively) (Table 1).

\subsection{Prescribed Daily Doses in 2017 versus 2007}

Table 2 shows the differences in doses in 2017 compared with 2007. For citalopram, escitalopram and mirtazapine, the mean doses in 2007 compared with 2017 were 20 vs. $17 \mathrm{mg} /$ day $(p<0.001), 11$ vs. $10 \mathrm{mg} /$ day $(p<0.001)$ and 28 vs. $25 \mathrm{mg} /$ day $(p=0.003)$, respectively. The most apparent difference was seen for venlafaxine, where a $17 \%$ dose reduction from 2007 to 2017 was observed ( 150 vs. $125 \mathrm{mg}$; $p<0.001)$. The mean sertraline dose was somewhat higher in 2017 compared with 2007, but the confidence intervals were large. Similar differences in mean prescribed doses between 2007 and 2017 were found for younger individuals (online resource 1).

\subsection{Measured Serum Concentrations in $\mathbf{2 0 1 7}$ versus 2007}

Mirtazapine was the only antidepressant where a significantly lower mean serum concentration was observed from 2007 to 2017 (50 vs. $41 \mathrm{ng} / \mathrm{mL} ; p<0.001$ ) (Table 3). For the other antidepressants, the observed dose reductions from 2007 to 2017 were not accompanied by concurrent reductions in mean serum concentration. On the contrary, for escitalopram, mean serum concentrations increased by $17 \%$ from 2007 to 2017 ( 18 vs. $21 \mathrm{ng} / \mathrm{mL} ; p=0.002)$ (Table 3). The differences in mean serum concentrations between 2007 and 2017 in younger individuals were similar to those observed in older individuals (online resource 2).

The use of multiple antidepressants was found in $20 \%$ of individuals aged $\geq 65$ years using selective serotonin 
Table 1 Characteristics for individuals included in the analyses of mean prescribed doses

\begin{tabular}{|c|c|c|c|c|c|}
\hline & Year & Age group, years & $\begin{array}{l}\text { Median age, years } \\
\text { (IQR) }\end{array}$ & $\begin{array}{l}\text { No. of individuals } \\
\text { (\% female) }\end{array}$ & $\begin{array}{l}\text { No. of samples } \\
\text { (\% female) }\end{array}$ \\
\hline \multirow[t]{5}{*}{ All antidepressants } & 2007 & $<65$ & $41(19)$ & $4409(60)$ & $5639(60)$ \\
\hline & & $\geq 65$ & $76(13)$ & $806(75)$ & $972(76)$ \\
\hline & 2017 & $<65$ & $41(22)$ & $4515(61)$ & $5604(62)$ \\
\hline & & $\geq 65$ & $79(15)$ & $1932(72)$ & $2441(72)$ \\
\hline & Total & & & $11,662(64)$ & $14,656(64)$ \\
\hline \multirow[t]{5}{*}{ Citalopram } & 2007 & $<65$ & $42(17)$ & $422(61)$ & $514(62)$ \\
\hline & & $\geq 65$ & 77 (13) & $126(75)$ & $141(74)$ \\
\hline & 2017 & $<65$ & $46(20)$ & $229(63)$ & $276(64)$ \\
\hline & & $\geq 65$ & $78(18)$ & $128(73)$ & $154(73)$ \\
\hline & Total & & & $905(65)$ & $1085(66)$ \\
\hline \multirow[t]{5}{*}{ Escitalopram } & 2007 & $<65$ & $41(20)$ & $2039(63)$ & $2594(64)$ \\
\hline & & $\geq 65$ & $75(12)$ & $268(74)$ & $320(76)$ \\
\hline & 2017 & $<65$ & $40(21)$ & $2216(64)$ & $2747(65)$ \\
\hline & & $\geq 65$ & $79(16)$ & $753(71)$ & $939(71)$ \\
\hline & Total & & & $5280(65)$ & $6600(66)$ \\
\hline \multirow[t]{5}{*}{ Sertraline } & 2007 & $<65$ & $39(19)$ & $456(64)$ & $599(64)$ \\
\hline & & $\geq 65$ & $78(13)$ & $92(83)$ & $111(81)$ \\
\hline & 2017 & $<65$ & $35(24)$ & $777(65)$ & $960(64)$ \\
\hline & & $\geq 65$ & $75(13)$ & $176(73)$ & $232(73)$ \\
\hline & Total & & & $1532(66)$ & $1938(65)$ \\
\hline \multirow[t]{5}{*}{ Mirtazapine } & 2007 & $<65$ & $44(19)$ & $452(50)$ & $591(51)$ \\
\hline & & $\geq 65$ & 77 (12) & $181(72)$ & $230(75)$ \\
\hline & 2017 & $<65$ & $46(22)$ & 435 (47) & $541(48)$ \\
\hline & & $\geq 65$ & $82(14)$ & $586(72)$ & 729 (72) \\
\hline & Total & & & $1654(59)$ & $2091(60)$ \\
\hline \multirow[t]{5}{*}{ Venlafaxine } & 2007 & $<65$ & 43 (18) & $1040(58)$ & $1341(56)$ \\
\hline & & $\geq 65$ & $72(12)$ & $139(74)$ & $170(74)$ \\
\hline & 2017 & $<65$ & $44(22)$ & 858 (59) & $1080(60)$ \\
\hline & & $\geq 65$ & 75 (13) & $289(74)$ & $387(73)$ \\
\hline & Total & & & $2326(61)$ & $2978(60)$ \\
\hline
\end{tabular}

$I Q R$ interquartile range

Table 2 Prescribed doses (mg/day) in individuals aged $\geq 65$ years, in 2017 versus 2007

\begin{tabular}{|c|c|c|c|c|}
\hline Antidepressant & & 2007 & 2017 & $p$ value $^{\mathrm{a}}$ \\
\hline \multirow[t]{2}{*}{ Citalopram } & Patients (samples) & $126(141)$ & $128(154)$ & \\
\hline & $\operatorname{Dose}^{b}$ & $20(19-22)$ & $17(16-18)$ & $<0.001$ \\
\hline \multirow[t]{2}{*}{ Escitalopram } & Patients (samples) & $268(320)$ & 753 (939) & \\
\hline & $\operatorname{Dose}^{b}$ & $11(11-12)$ & $10(10-10)$ & $<0.001$ \\
\hline \multirow[t]{2}{*}{ Sertraline } & Patients (samples) & $92(111)$ & $176(232)$ & \\
\hline & $\operatorname{Dose}^{b}$ & $60(54-67)$ & 72 (67-77) & 0.10 \\
\hline \multirow[t]{2}{*}{ Mirtazapine } & Patients (samples) & $181(230)$ & $586(729)$ & \\
\hline & Dose $^{b}$ & $28(27-30)$ & $25(25-26)$ & 0.003 \\
\hline \multirow[t]{2}{*}{ Venlafaxine } & Patients (samples) & $139(170)$ & $289(387)$ & \\
\hline & $\operatorname{Dose}^{b}$ & $150(139-163)$ & $125(118-133)$ & $<0.001$ \\
\hline
\end{tabular}

${ }^{\mathrm{a}}$ By linear mixed-effects model, adjusted for sex

${ }^{\mathrm{b}}$ Data are expressed as geometric mean (95\% confidence interval) 
reuptake inhibitors (SSRIs) or venlafaxine in 2017, which was twofold higher when compared with younger individuals $(p=0.001)$ [online resource 3]. For mirtazapine, the proportion was higher in both younger and older individuals (47\% and 40\%, respectively) [online resource 3]. The findings for 2007 were comparable (data not shown).

\subsection{Proportions Above the Reference Range in $\mathbf{2 0 1 7}$ versus 2007}

A lower proportion of individuals with at least one serum concentration above the recommended reference range was only found for mirtazapine (27.1 vs. $11.5 \% ; p<0.001)$, while a tendency for a reduction was seen for venlafaxine ( 42.9 vs. $34.5 \%$; $p=0.12$ ) (Fig. 2 a). However, a significant reduction from 2007 to 2017 was observed for individuals aged $\geq 80$ years using venlafaxine $(60.0 \%$ vs. $30.0 \% ; p=0.007$ ) (Fig. 2c). No difference in the proportion above the recommended reference range in 2017 compared with 2007 was seen for citalopram, escitalopram or sertraline, or when differentiating between individuals aged $65-79$ years and individuals aged $\geq 80$ years (Fig. 2). There were no sex differences, except for citalopram, where the proportion with serum concentrations above the recommended reference range was significantly higher for women compared with men (15.4\% vs. $1.8 \%)$ [data not shown].

Corresponding findings for younger individuals are shown in online resource 4. In both 2007 and 2017, the proportion of individuals with at least one serum concentration above the recommended reference range was almost doubled among older individuals compared with younger individuals (16.8\% vs. $9.0 \%$ in 2007 and $10.6 \%$ vs. $6.0 \%$ in 2017) (Fig. 2a in the main text, and online resource 4).

\section{Discussion}

In the present study examining data from a TDM service in Norway in 2007 and 2017, we observed minor reductions in the prescribed doses of antidepressants to older people. Despite statistically significant differences, the observed reductions were small, especially for the most commonly used antidepressants citalopram and escitalopram. For mirtazapine, the dose reduction was accompanied by a corresponding reduction in the proportion of individuals with serum concentrations above the recommended reference range; this was also observed for individuals aged $\geq 80$ years using venlafaxine, but not for the commonly used citalopram and escitalopram. Similar changes from 2007 to 2017 were also observed for younger individuals ( $<65$ years), indicating that the observed reductions are not specifically associated with old age.

The majority of the TDM data analyzed in this study were from patients using escitalopram, which is the antidepressant most often used among both older and younger individuals in Norway [2]. The finding of reductions in prescribed doses of escitalopram and citalopram complies with the regulatory warnings and lower recommended doses at the end of 2011, although mean prescribed doses in 2017 are still close to the maximum recommended doses [24, 25]. The recommendation on reducing the maximum doses of citalopram and escitalopram was based on reports of a correlation between the administered dose and the risk of QTc prolongation [24, 25]. Although the literature is sparse regarding the direct association between serum concentrations of SSRIs and the risk of cardiac toxicity, a correlation between QTc prolongation and serum concentrations of citalopram after intoxication has been reported [27]. Overall, the higher proportions of older individuals with serum concentrations above the

Table 3 Serum concentrations (ng/mL) in individuals aged $\geq 65$ years, in 2017 versus 2007

\begin{tabular}{|c|c|c|c|c|}
\hline Antidepressant & & 2007 & 2017 & $p$ value $^{\mathrm{a}}$ \\
\hline \multirow[t]{2}{*}{ Citalopram } & Patients (samples) & 104 (116) & 115 (139) & \\
\hline & Serum concentration $^{\mathrm{b}}$ & $55(48-62)$ & $49(44-54)$ & 0.15 \\
\hline \multirow[t]{2}{*}{ Escitalopram } & Patients (samples) & $225(266)$ & $693(858)$ & \\
\hline & Serum concentration ${ }^{\mathrm{b}}$ & $18(17-20)$ & $21(20-22)$ & 0.002 \\
\hline \multirow[t]{2}{*}{ Sertraline } & Patients (samples) & $78(95)$ & $164(214)$ & \\
\hline & Serum concentration $^{\mathrm{b}}$ & $20(17-24)$ & $24(21-26)$ & 0.77 \\
\hline \multirow[t]{2}{*}{ Mirtazapine } & Patients (samples) & $167(208)$ & $565(695)$ & \\
\hline & Serum concentration ${ }^{\mathrm{b}}$ & $50(45-54)$ & $41(39-43)$ & 0.001 \\
\hline \multirow[t]{2}{*}{ Venlafaxine } & Patients (samples) & $119(148)$ & $261(344)$ & \\
\hline & Serum concentration ${ }^{\mathrm{b}}$ & $340(303-381)$ & $310(292-329)$ & 0.46 \\
\hline
\end{tabular}

${ }^{a}$ By linear mixed-effects model, adjusted for sex and sampling time

${ }^{b}$ Data are expressed as geometric mean (95\% confidence interval) 


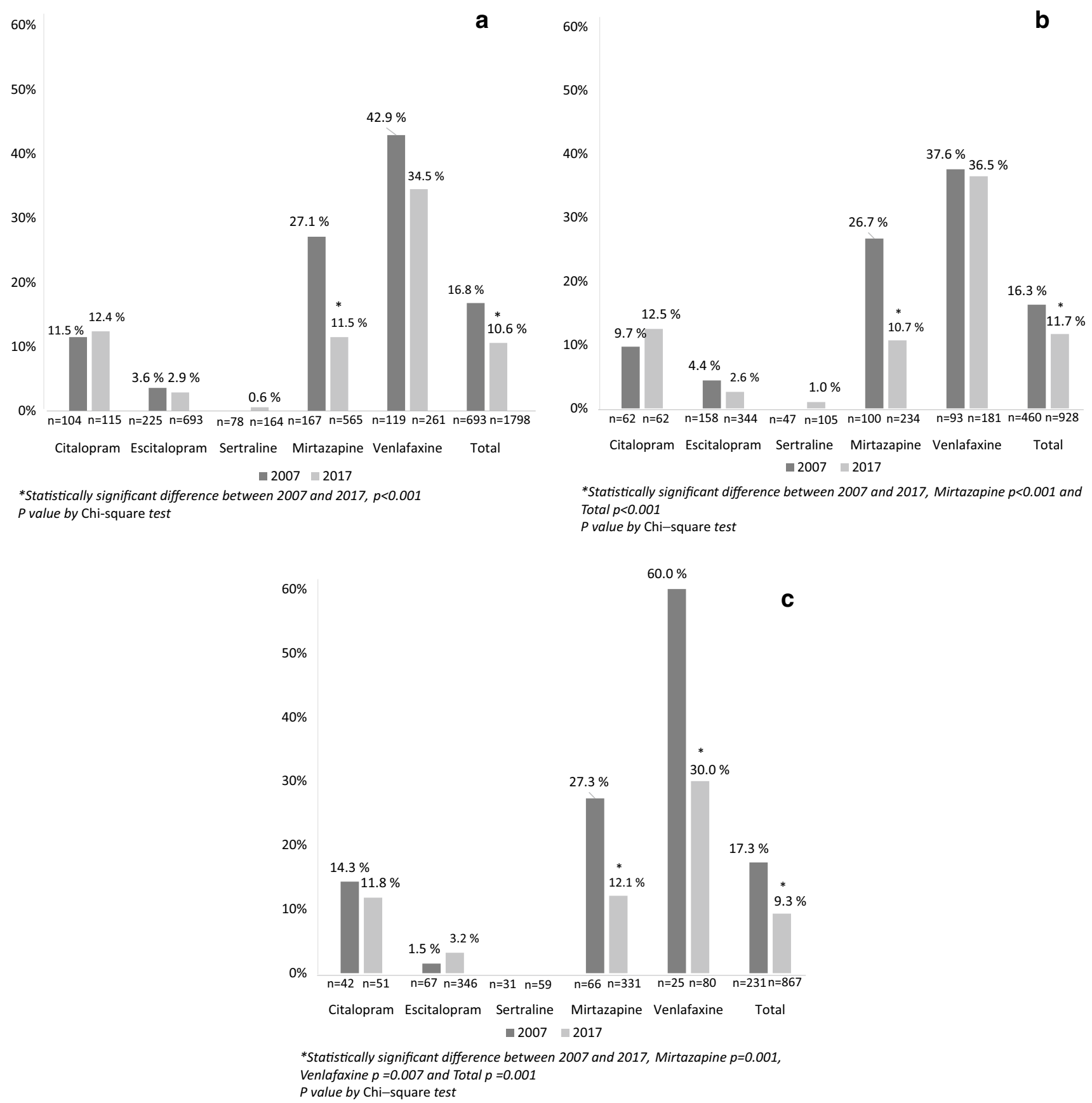

Fig. 2 Fraction of individuals with at least one sample above the recommended reference range. $\mathbf{a} \geq 65$ years; $\mathbf{b} 65-79$ years; $\mathbf{c} \geq 80$ years

recommended reference range may indicate a higher risk of cardiac adverse effects in these individuals.

A clear reduction in both prescribed doses, mean serum concentration and the proportion of patients with serum concentrations above the recommended reference range was observed for mirtazapine. Mirtazapine is an atypical antidepressant with a considerable sedative effect prominent in low-dose regimens, and, because of its distinctive receptor profile and anticipated lower risk of adverse drug reactions such as hyponatremia and bleeding, mirtazapine is a frequently used comedication with other antidepressants and might also be a preferred antidepressant in patients with considerable comorbidity and polypharmacy [28-31]. The prominent reduction in the proportion of individuals with serum concentrations above the recommended reference range observed in our study may imply a reduced risk of adverse effects in these patients. However, the lower doses and serum concentrations of this substance found in 2017, 
compared with 2007, may be due to increased use as an add-on therapy and/or increased use as a sedative, perhaps because of changes in regulatory warnings on the use of antipsychotics to older individuals. Furthermore, in our study, $40 \%$ of older individuals treated with mirtazapine had additional antidepressants detected in the blood in 2017, which was twice the frequency observed for individuals treated with any of the other antidepressants.

Venlafaxine displayed the greatest reduction in prescribed doses between 2007 and 2017, and also an overall tendency of a reduction in the proportion of individuals aged $\geq 65$ years with serum concentrations above the recommended reference range. However, when examining differences within the older individuals specifically, the proportion of individuals with serum concentrations above the recommended reference range between 2007 and 2017 was reduced by $50 \%$ in individuals $\geq 80$ years, while no change was observed for individuals aged 65-79 years. The dose reduction observed in the oldest patients could possibly be explained by the increased focus on adverse effects associated with higher doses of venlafaxine [32]. Recently, an association between QTc prolongation and high serum concentration was demonstrated for older people using venlafaxine [33]. Increased awareness about the age-related higher serum concentrations in older individuals treated with venlafaxine could be another explanation, but reductions in prescribed doses between 2007 and 2017 in individuals aged $<65$ years were similar, indicating that age-related changes in pharmacokinetics and pharmacodynamics are not the reason for the observed dose reduction [19-21]. In our study, the proportion of patients with serum concentrations of venlafaxine above the recommended reference range was high, which may partly be explained by a difference between the reference range used locally at Diakonhjemmet Hospital, where the TDM analysis were performed, and the reference range recommended by the AGNP.

In spite of the small reduction in the mean prescribed dose for escitalopram, the mean serum concentration actually increased between 2007 and 2017. This implies an increase in the dose-adjusted serum concentration (C/D ratio) between 2007 and 2017; when the C/D ratio was investigated, a similar pattern was also observed for venlafaxine. Because of gradual changes in pharmacokinetics with increased age, treatment of a relatively older population, as shown by a higher median age in our study, would possibly contribute to the increased C/D ratio. However, age cannot be the only explanation since a corresponding pattern was also observed among younger individuals where the median age was similar between 2007 and 2017. Another factor contributing to the increased C/D ratio may be the increased comedication with interacting drugs, e.g. proton pump inhibitors inhibiting the cytochrome P450 (CYP) 2C19 metabolism. However, due to the limited and unstructured information about comedications on the TDM requisition forms, this speculation cannot be further elucidated. Furthermore, a general increased attention towards adverse drug reactions of antidepressant medications could possibly have resulted in a change in the TDM requisition pattern where clinicians, to an increased extent, may request serum concentration measurements when high values are expected.

In our study, the higher proportion of TDM samples from older individuals in 2017 compared with 2007 might be interpreted as improved follow-up of antidepressant therapy in older individuals, possibly as a result of an increased focus on drug safety and follow-up of older patients, and more knowledge on adverse drug reactions [22-25]. On the other hand, only modest reductions in prescribed doses and serum concentration measurements of antidepressants in older individuals were observed when compared with younger individuals. For all five antidepressants, the proportion of individuals with serum concentrations above the recommended reference range was still near twofold higher among older individuals compared with younger individuals in 2017, which is comparable with earlier findings [22]. With the exception of venlafaxine, no differences between 2007 and 2017 in the proportion of individuals with serum concentrations above the recommended reference range were found when comparing people within the older patient group (65-79 years vs. $\geq 80$ years). In addition, in 2017, the use of multiple antidepressants was twofold higher in older individuals, with the exception of mirtazapine. In total, our findings indicate that older individuals are more intensively treated when compared with younger individuals.

It must be noted that serum concentrations above the recommended reference range does not necessarily mean overmedication, as the TDM reference ranges are not absolutely defined therapeutic ranges. In addition, specific TDM reference ranges adjusted to older and frail patients do not exist [26]. The higher serum levels among older individuals observed in the present study does not necessarily mean that older individuals are overmedicated; on the contrary, it could indicate that there are several younger patients treated with suboptimal antidepressant doses. Furthermore, a higher risk may be justified if treatment response is improved. Nevertheless, there is increasing evidence on both the dose-dependent effect of antidepressants and between serum levels and clinical improvement/adverse effects, including no increased effect when the recommended reference ranges are exceeded [27, 33-38]. A recent work from our laboratory demonstrated a u-shaped risk of switching from escitalopram to alternative antidepressant therapy that correlated with both high and low serum concentrations of escitalopram, which likely reflects unacceptable adverse effects or lack of effect, respectively [38].

When using TDM data, there are some limitations that should be taken into consideration. A possible bias is that 
TDM may be performed more frequently when non-compliance or adverse drug reactions are suspected, which might also be different between the two age groups [39-43]. In addition, it can be assumed that TDM is requested more frequently when the total treatment load is high, i.e. when patients are using high doses or multiple antidepressant and/ or additional psychotropic drugs simultaneously. Furthermore, antidepressant doses may differ between diagnosis and the duration of treatment, as careful dose adjustments are recommended in the initiation and termination of antidepressant therapy. Unfortunately, in our study, information on the reasons for TDM was not available, neither on diagnosis, use of additional psychotropic drugs, nor duration of use. Finally, use of antidepressants may differ between primary and secondary health care, for which we had only limited information. On the other hand, TDM data make it possible to consider serum concentrations, and samples where medication has not been taken (i.e. no antidepressant detected) can be identified. In Norway, the costs for the use of TDM are covered by the publicly financed health care system, which imply that decisions on the use of TDM are not limited by the financial situation of the patient. Furthermore, the large sample size from both primary and specialist health care, and from all parts of the country, contributes to important knowledge regarding changes in antidepressant treatment in older individuals.

\section{Conclusion}

During the last decade, there has generally been a small reduction in the prescribed doses of antidepressants among older individuals in Norway. A reduction in the proportion of older individuals with serum concentrations above the recommended reference range was only found for mirtazapine and individuals aged $\geq 80$ years using venlafaxine. For the most commonly used antidepressants citalopram and escitalopram, prescribed doses have been slightly reduced (10-15\%), but the proportion of patients with serum concentrations above the recommended reference range has not changed. The overall findings of this study suggest that a considerable proportion of older individuals still attain high serum concentrations of antidepressant medications.

Acknowledgements Open Access funding provided by Western Norway University Of Applied Sciences.

Author Contributions $\mathrm{MH}$ and EM initiated and designed the project, with contributions from SMW and KT. RBW extracted the data from the TDM database. Data processing and statistical analysis were performed by KT, with contributions from RMN and RBW. KT was responsible for data interpretation, with contributions from MH, SMW and EM. KT drafted the manuscript, with contributions from RBW and RMN. MH, SMW and EM reviewed the manuscript for important intellectual content. All authors read and approved the final version of the manuscript.

\section{Compliance with Ethical Standards}

Funding Authors own institutions.

Conflicts of interest Kristine Tveit, Monica Hermann, Ragnhild Birkeland Waade, Roy Miodini Nilsen, Susanna M. Wallerstedt and Espen Molden declare they have no conflicts of interest.

Ethics approval This study was approved by the Regional Committee for Medical and Health Research Ethics (ref. 2018/655) and the Hospital Investigational Review Board.

Consent to participate Ethical approval was granted without the collection of informed consent from participants. This was based on the evaluation of risk-benefit and also practical reasons (historical data from more than 17,000 individuals). To protect confidentiality, personal identifiers were not included in the dataset.

Data availability The datasets generated and analyzed during the current study are available from the corresponding author upon reasonable request.

Open Access This article is licensed under a Creative Commons Attribution-NonCommercial 4.0 International License, which permits any non-commercial use, sharing, adaptation, distribution and reproduction in any medium or format, as long as you give appropriate credit to the original author(s) and the source, provide a link to the Creative Commons licence, and indicate if changes were made. The images or other third party material in this article are included in the article's Creative Commons licence, unless indicated otherwise in a credit line to the material. If material is not included in the article's Creative Commons licence and your intended use is not permitted by statutory regulation or exceeds the permitted use, you will need to obtain permission directly from the copyright holder. To view a copy of this licence, visit http://creativecommons.org/licenses/by-nc/4.0/.

\section{References}

1. Kok RM, Reynolds CF. Management of depression in older adults: a review. JAMA. 2017;317(20):2114-222.

2. Norwegian Prescription Database. Norwegian Institute of Public Health. 2019. https://www.reseptregisteret.no/. Accessed 25 Sep 2019.

3. Drug Consumption in Norway 2012-2016. Norwegian Institute of Public Health. 2017. https://www.fhi.no/contentassets/0a8afbeb4c 904d14b7b8a773bc25766e/legemiddelforbruket-2012-2016.pdf. Accessed 15 Mar 2017.

4. Hansen DG, Rosholm J-U, Gichangi A, Vach W. Increased use of antidepressants at the end of life: population-based study among people aged 65 years and above. Age Ageing. 2007;36(4):449-54.

5. Health at a Glance 2019. OECD. 2019. https://www.oecdilibrary.org/social-issues-migration-health/health-at-a-glanc e-2019_4dd50c09-en. Accessed 4 Jun 2020.

6. Winerman L. By the numbers: Antidepressant use on the rise. In: Monitor on Psychology. American Psychological Association. 2017. https://www.apa.org/monitor/2017/11/numbers. Accessed 4 Jun 2020 . 
7. Darowski A, Chambers SA, Chambers DJ. Antidepressants and falls in the elderly. Drugs Aging. 2009;26(5):381-94. https://doi. org/10.2165/00002512-200926050-00002.

8. Gandhi S, Shariff SZ, Al-Jaishi A, Reiss JP, Mamdani MM, Hackam DG, et al. Second-generation antidepressants and hyponatremia risk: a population-based cohort study of older adults. Am J Kidney Dis. 2017;69(1):87-96.

9. Read J, Williams J. Adverse effects of antidepressants reported by a large international cohort: emotional blunting, suicidality, and withdrawal effects. Curr Drug Saf. 2018;13(3):176-86. https ://doi.org/10.2174/1574886313666180605095130.

10. Mangoni AA, Jackson SH. Age-related changes in pharmacokinetics and pharmacodynamics: basic principles and practical applications. Br J Clin Pharmacol. 2004;57(1):6-14.

11. Davies EA, O'Mahony MS. Adverse drug reactions in special populations-the elderly. Br J Clin Pharmacol. 2015;80(4):796-807.

12. Ćurković M, Dodig-Ćurković K, Petek Erić A, Kralik K, Pivac N. Psychotropic medications in older adults: a review. Psychiatria Danubina. 2016;28(1):1-24.

13. O'Mahony D, O'Sullivan D, Byrne S, O'Connor MN, Ryan C, Gallagher P. STOPP/START criteria for potentially inappropriate prescribing in older people: version 2. Age Ageing. 2015;44(2):213-8.

14. Hill KD, Wee R. Psychotropic drug-induced falls in older people: a review of interventions aimed at reducing the problem. Drugs Aging. 2012;29(1):15-30. https://doi.org/10.2165/11598420000000000-00000.

15. Johnell K, Klarin I. The relationship between number of drugs and potential drug-drug interactions in the elderly: a study of over 600,000 elderly patients from the Swedish Prescribed Drug Register. Drug Saf. 2007;30(10):911-8. https://doi.org/10.2165/00002 018-200730100-00009.

16. Petrovic M, van der Cammen T, Onder G. Adverse drug reactions in older people: detection and prevention. Drugs Aging. 2012;29(6):453-62. https://doi.org/10.2165/11631760-00000 $0000-00000$

17. Rockwood K, Howlett SE. Age-related deficit accumulation and the diseases of ageing. Mech Ageing Dev. 2019;180:107-16. https ://doi.org/10.1016/j.mad.2019.04.005.

18. Waade RB, Molden E, Refsum H, Hermann M. Serum concentrations of antidepressants in the elderly. Ther Drug Monit. 2012;34(1):25-30.

19. Waade RB, Hermann M, Moe HL, Molden E. Impact of age on serum concentrations of venlafaxine and escitalopram in different CYP2D6 and CYP2C19 genotype subgroups. Eur J Clin Pharmacol. 2014;70(8):933-40. https://doi.org/10.1007/s0022 8-014-1696-8.

20. Hansen MR, Kuhlmann IB, Pottegård A, Damkier P. Therapeutic drug monitoring of venlafaxine in an everyday clinical setting: analysis of age, sex and dose concentration relationships. Basic Clin Pharmacol Toxicol. 2017;121(4):298-302.

21. Sigurdsson HP, Hefner G, Ben-Omar N, Köstlbacher A, WenzelSeifert K, Hiemke C, et al. Steady-state serum concentrations of venlafaxine in patients with late-life depression. Impact of age, sex and BMI. J Neural Transm. 2015;122(5):721-9.

22. Hermann M, Waade RB, Molden E. Therapeutic drug monitoring of selective serotonin reuptake inhibitors in elderly patients. Ther Drug Monit. 2015;37(4):546-9. https://doi.org/10.1097/ FTD.0000000000000169.

23. The Norwegian Patient Safety Programme: In Safe Hands. Norwegian Ministry of Health Care Services. 2016. https://www.pasie ntsikkerhetsprogrammet.no/om-oss/english/the-norwegian-patie nt-safety-programme-in-safe-hands. Accessed 25 Sep 2019.

24. Safety concerns: Citalopram-Risk of QT interval prolongation. In: Monthly report: 1110. European Medicines Agency. 2011. https://www.ema.europa.eu/en/documents/report/monthly-repor t-pharmacovigilance-working-party-phvwp-october-2011-plena ry-meeting_en.pdf. Accessed 15 Mar 2019.

25. Safety concerns: Escitalopram-Risk of QT interval prolongation. In: Monthly report: 1111. European Medicines Agency. 2011. https://www.ema.europa.eu/en/documents/report/monthly-repor t-pharmacovigilance-working-party-phvwp-november-2011-plena ry-meeting_en.pdf. Accessed 15 Mar 2019.

26. Hiemke C, Bergemann N, Clement HW, Conca A, Deckert J, Domschke K, et al. Consensus guidelines for therapeutic drug monitoring in neuropsychopharmacology: update 2017. Pharmacopsychiatry. 2018;51(01/02):9-62.

27. Unterecker S, Warrings B, Deckert J, Pfuhlmann B. Correlation of QTc interval prolongation and serum level of citalopram after intoxication-a case report. Pharmacopsychiatry. 2012;45(01):30-4.

28. Mirtazapine $15 \mathrm{mg}$ orodispersible tablets. EMC. 2019. https:// www.medicines.org.uk/emc/product/4750/smpc. Accessed 15 Mar 2019.

29. Watanabe N, Omori IM, Nakagawa A, Cipriani A, Barbui C, Churchill R, et al. Mirtazapine versus other antidepressive agents for depression. Cochrane Database Syst Rev. 2011;12:CD006528.

30. Bixby AL, VandenBerg A, Bostwick JR. Clinical management of bleeding risk with antidepressants. Ann Pharmacother. 2019;53(2):186-94.

31. Ionescu DF, Rosenbaum JF, Alpert JE. Pharmacological approaches to the challenge of treatment-resistant depression. Dialogues Clin Neurosci. 2015;17(2):111.

32. Shelton RC. Serotonin and norepinephrine reuptake inhibitors. Handb Exp Pharmacol. 2019;250:145-80. https://doi. org/10.1007/164_2018_164.

33. Hefner G, Hahn M, Hohner M, Roll SC, Klimke A, Hiemke C. QTC time correlates with amitriptyline and venlafaxine serum levels in elderly psychiatric inpatients. Pharmacopsychiatry. 2019;52(01):38-433.

34. Hieronymus F, Nilsson S, Eriksson E. A mega-analysis of fixeddose trials reveals dose-dependency and a rapid onset of action for the antidepressant effect of three selective serotonin reuptake inhibitors. Transl Psychiatry. 2016;6(6):e834. https://doi. org/10.1038/tp.2016.104.

35. Furukawa TA, Cipriani A, Cowen PJ, Leucht S, Egger M, Salanti G. Optimal dose of selective serotonin reuptake inhibitors, venlafaxine, and mirtazapine in major depression: a systematic review and doseresponse meta-analysis. Lancet Psychiatry. 2019;6(7):601-9. https ://doi.org/10.1016/s2215-0366(19)30217-2.

36. Haji EO, Tadic A, Wagner S, Dragicevic A, Müller MJ, Boland $\mathrm{K}$, et al. Association between citalopram serum levels and clinical improvement of patients with major depression. J Clin Psychopharmacol. 2011;31(3):281-6.

37. Florio V, Porcelli S, Saria A, Serretti A, Conca A. Escitalopram plasma levels and antidepressant response. Eur Neuropsychopharmacol. 2017;27(9):940-4.

38. Jukić MM, Haslemo T, Molden E, Ingelman-Sundberg M. Impact of CYP2C19 genotype on escitalopram exposure and therapeutic failure: a retrospective study based on 2,087 patients. Am J Psychiatry. 2018;175(5):463-70.

39. Barat I, Andreasen F, Damsgaard EM. Drug therapy in the elderly: what doctors believe and patients actually do. Br J Clin Pharmacol. 2001;51(6):615-22. https://doi.org/10.104 6/j.0306-5251.2001.01401.x.

40. Tveito M, Bramness JG, Engedal K, Lorentzen B, Refsum H, Hoiseth G. Psychotropic medication in geriatric psychiatric patients: use and unreported use in relation to serum concentrations. Eur J Clin Pharmacol. 2014;70(9):1139-45. https://doi.org/10.1007/ s00228-014-1720-z.

41. Leven EA, Annunziato R, Helcer J, Lieber SR, Knight CS, Wlodarkiewicz C, et al. Medication adherence and rejection rates 
in older vs younger adult liver transplant recipients. Clin Transplant. 2017;31(6):e12981.

42. Range JT, LaFontaine PR, Ryder PT, Polston M. Factors associated with adherence to statin medications of patients enrolled in a self-insured university health plan. Clin Ther. 2018;40(10):1692700. https://doi.org/10.1016/j.clinthera.2018.08.012.
43. Pasina L, Brucato AL, Falcone C, Cucchi E, Bresciani A, Sottocorno $\mathrm{M}$, et al. Medication non-adherence among elderly patients newly discharged and receiving polypharmacy. Drugs Aging. 2014;31(4):283-9. https://doi.org/10.1007/s40266-014-0163-7.

\section{Affiliations}

\section{Kristine Tveit $^{1}\left({ }^{10} \cdot\right.$ Monica Hermann $^{1} \cdot$ Ragnhild Birkeland Waade $^{2}(1) \cdot$ Roy Miodini Nilsen $^{1}$.}

\section{Susanna M. Wallerstedt ${ }^{3,4}$ (D) Espen Molden ${ }^{2,5}$}

1 Department of Health and Caring Sciences, Western Norway University of Applied Sciences, Bergen, Haugesund, Stord, Norway

2 Center for Psychopharmacology, Diakonhjemmet Hospital, Oslo, Norway
3 Department of Pharmacology, Sahlgrenska Academy, University of Gothenburg, Gothenburg, Sweden

4 Sahlgrenska University Hospital, Gothenburg, Sweden

5 Department of Pharmacy, University of Oslo, Oslo, Norway 